\title{
FREE-CARRIER PLASMONS AS A NOVEL TOOL IN SEMICONDUCTOR PHYSICS*
}

\author{
W. SZuszkiewicz, \\ Institute of Experimental Physics, Warsaw University, Hoża 69, 00-681 Warszawa, \\ Poland \\ W. BARDYSZEWSKI, \\ Institute of Theoretical Physics, Warsaw University, Warsaw, Poland
}

QIan Dingrong, Zhang Jiaming,

Shanghai Institute of Technical Physics, Academia Sinica, Shanghai, China

$$
\text { C. Julien, M. Balkanski, }
$$

Laboratoire de Physique des Solides, Université Paris VI, Paris, France

\section{B. Witkowska aNd A. Mycielski}

Institute of Physics, Polish Academy of Sciences, Warsaw, Poland

(Received August 8, 1990)

\begin{abstract}
It is demonstrated that free-carrier plasmons, being well defined collective excitations of the electron gas in the range of small wave vectors, can serve as a sensitive tool to investigate the optical processes related to the small momentum transfers. As an example the system $\mathrm{HgSe:Fe}$ is analysed both experimentally and theoretically.
\end{abstract}

PACS numbers: 71.45.Gm, 72.30.+q, 75.50.Pp

It is well known that the excitation of the free-carrier plasma in the light absorption process is possible only in the presence of defects breaking the translational invariance of the system. Due to the overall momentum conservation requirement there must exist a momentum source to make the photon absorption

*This work is supported in part by CPBP 01.06. 
accompanied by plasmon generation possible (see [1] and the references therein). The simplest case occurs when there are ionized impurities in the system. The corre-

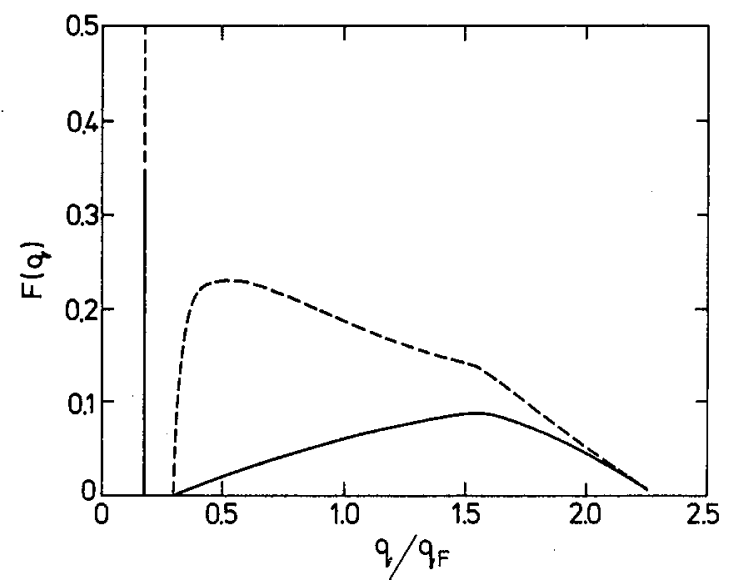

Fig. 1. The function $F(q)$ for HgSe:Fe at $T=5 \mathrm{~K}$ for the energy slightly greater than the plasma energy. The iron concentration is equal to about $N_{\mathrm{Fe}}=4 \times 10^{19}$ $\mathrm{cm}^{-3}$, the electron concentration $N=4.5 \times 10^{18} \mathrm{~cm}^{-3}$ (equal to the ionized impurity concentration) has been assumed. The broken line corresponds to the theoretical value of $F(q)$ calculated assuming the random distribution of the ionized impurities. The solid line corresponds to the ordering of ionized impurities predicted by the theory given in $[6,7]$. The delta peak well seen for the small $q$ values corresponds to the collective plasma excitations. The contribution to the inverse relaxation time related to this peak is of the order of $30 \%$ of the total value for the random distribution of the ionized impurities. This contribution is reduced about 100 times when the ordering of the ionized impurities is taken into account.

sponding absorption coefficient is proportional to the structure factor of impurities which is determined by their distribution in the solid [2]. When the positions of ionized centers are entirely random this factor does not depend on the momentum transfer and therefore it does not favour any particular type of the electron gas excitations. However, if there exists any kind of ordering in the impurity positions the excitations with certain momenta are less likely generated. As a consequence one should observe a reduction of the role of associated inelastic processes in the light absorption. This effect is especially important for small momentum transfers where plasmons are dominant excitations responsible for the light absorption. Thus if some ordering in the system of ionized impurities exists it can be detected by the plasmon related free-carrier absorption.

As an example we study both experimentally and theoretically $\mathrm{HgSe}: \mathrm{Fe}$ in which at low temperatures for iron concentration higher than about $N_{\mathrm{Fe}}=5 \times 10^{18}$ $\mathrm{cm}^{-3}$ the superlattice of charged impurity centers might be created [3-5]. In such a case we would have a total suppresion of the scattering amplitude for electron 


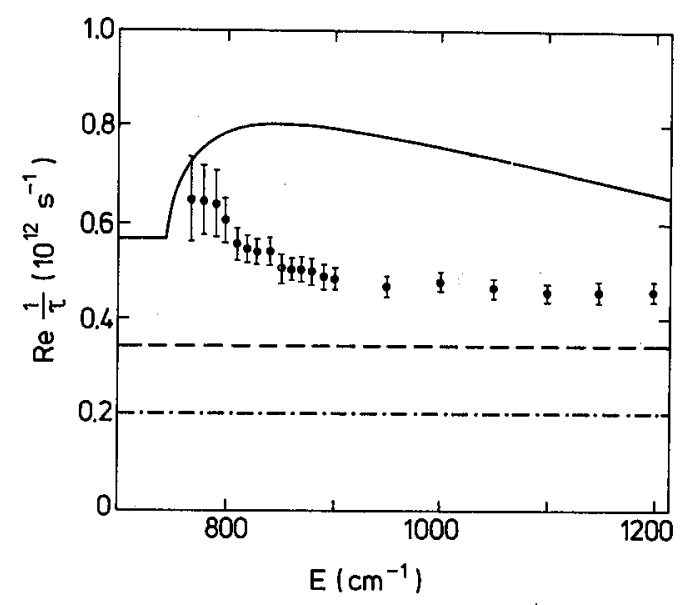

Fig. 2. The real part of the inverse relaxation time determined experimentally and theoretically for the sample with $N_{\mathrm{Fe}}=4 \times 10^{19} \mathrm{~cm}^{-3}$ at $T=5 \mathrm{~K}$. Dashed-dotted line the contribution due to the optical phonon term, broken line - the sum of the previous term and the contribution due to the scattering on the ionized impurities (random distribution), solid line - the sum of the phonon term and the ionized impurity term (assuming correlation given by $[6,7]$ ). The experimental points result from the reflectivity and transmission measurements taken at this temperature. The structure seen in the low-energy part of the experimental inverse relaxation time is attributed to the tail of the photoionisation contribution (the optical transitions from the occupied iron $\mathrm{Fe}^{2+}$ states to the empty conduction band states lying above the Fermi level).

gas excitations except the momenta corresponding to the Bragg condition. When the short-range correlation in the positions of the ionized impurities is assumed $[6,7]$, the electron gas excitations are strongly suppresed for the relatively small momenta values.

The real part of the inverse relaxation time for the scattering on the ionized impurities is given by the formula:

$$
\operatorname{Re}(1 / \tau)=\int_{0}^{2 k_{F}} F(q) S(q) d q
$$

where $S(q)$ is the structure factor, equal to 1 for the entirely random positions of the ionized centers. Figure 1. shows an example of the function $F(q)$, calculated for the iron concentration $N_{\mathrm{Fe}}=4 \times 10^{19} \mathrm{~cm}^{-3}$ as well as the product of $F(q)$ and $S(q)$, corresponding to the correlation resulting from the model given in $[6,7]$. As one can see due to the ordering of the charged centers the plasmon-related contribution to the relaxation time should be in practice totally suppressed. We have measured both the transmission and reflectivity spectra in the vicinity of the plasma frequency for $\mathrm{HgSe}: \mathrm{Fe}$ samples with small and large concentrations of $\mathrm{Fe}$ atoms at different temperatures. On the basis of the experimental data the real 
part of the inverse relaxation time has been determined. At room temperature the results obtained are not far from the values predicted by the theory taking into account a completely random distribution of ionized centers (one should expect some difference due to the high nonparabolicity of the $\mathrm{HgSe}$ conduction band at this temperature whereas the applied theory $[2,8]$ corresponds to the parabolic conduction band). The experimental data shows some suppresion of the inverse relaxation time with the decreasing temperature and, in particular, the strong suppresion of the plasmon related structure. As an example the comparison of the experimental and theoretical values of the inverse relaxation time for one of our samples at low temperature is shown in Fig. 2. In conclusion we can state that plasmons, being well defined collective excitations of the electron gas in the range of small wave vectors, can serve as a sensitive tool in studying the position correlation of Coulomb centers.

Authors would like to express their gratitude to Prof.J. Blinowski and Prof.M. Grynberg for helpful comments and discussions. One of us (W.Sz) wishes to thank Université Pierre et Marie Curie for the financial support during his stay in Paris.

\section{References}

[1] J. Mycielski, A. Mycielski, Phys.Rev. B 18, 1859 (1978).

[2] S. Goettig, J.Phys.C, Solid State Phys. 17, 4443 (1984).

[3] J. Mycielski, Solid State Commun. 60, 165 (1986).

[4] A. Mycielski, B.Kowalski, B. A. Orłowski, M. Dobrowolska, M. Arciszewska, W. Dobrowolski, J. M. Baranowski, J.Phys. C 19, 3605 (1986).

[5] F.S. Pool, J. Kossut, U. Dębska, R. Reifenberger, Phys. Rev. B 35, 3900 (1987).

[6] J. Kossut, W. Dobrowolski, Z. Wilamowski, T. Dietl, K. Świątek, Semicond. Sci. Technol. in press.

[7] Z. Wilamowski, K. Świątek, T. Dietl, J. Kossut, J. Vac. Sci. Technol. in press.

[8] S. Goettig, Solid State Commun. 55, 611 (1985). 